\title{
EL PARADIGMA HEGEMÓNICO MASCULINO: IMPLICACIONES PARA LA POLÍTICA Y LA ADMINISTRACIÓN PÚBLICA
}

\author{
Irvyn E. Nieves-Rolón ${ }^{I}$ \\ Víctor I. García-Toro ${ }^{2}$
}

\section{Resumen}

Este artículo elabora una reflexión crítica en torno a las implicaciones de los requerimientos del discurso de la masculinidad hegemónica en los procesos de elaboración de política pública. Se argumenta en términos de cuestionar, desde esta perspectiva, la gobernanza y la efectividad del proceso político sobre los géneros y la ciudadanía. Se cuestionan seriamente las acciones a tomar para lidiar con el paradigma hegemónico masculino, dado su impacto en un proceso político que debe procurar el bienestar y la equidad entre los géneros.

Descriptores: género masculino, género femenino, paradigma, masculinidad hegemónica, política pública y gobernanza.

\begin{abstract}
This article develops a critical reflection on the implications of the requirements of the discourse of hegemonic masculinity in the process of developing public policy. It is argued in terms of questioning from this perspective, effective governance and the political process on gender and citizenship. It also questions the actions taken to deal with the paradigm of male hegemony, given the impact in a political process that should ensure welfare and gender equity.
\end{abstract}

Keywords: male, female, paradigm, hegemonic masculinity, public policy and governance

\section{Introducción}

Los relatos de poder desde las esferas de lo económico, político y social, han marcado las pautas de los acontecimientos sociales a lo largo de la historia. Parte de las ideas fusionadas a esos esquemas institucionalizados de poder, constituyen el paradigma de la supremacía de lo masculino. Dicho esquema pauta códigos de conducta según el sexo y asigna al hombre el "deber" de regirse por la heterosexualidad, el control la provisión de recursos, la fuerza y la competencia (Ramírez y García Toro, 2002). En el esfuerzo de estudiar las características y situaciones relacionadas a las masculinidades, debemos reflexionar sobre cómo éstas afectan el desarrollo de política pública y la administración pública.

\footnotetext{
1 Profesor Departamento de Trabajo Social, Escuela de Ciencias Sociales y Humanas, Universidad del Este y Estudiante Doctoral en la Escuela Graduada de Trabajo Social Beatriz Lassalle, Universidad de Puerto Rico en Río Piedras

2 Catedrático Jubilado, Escuela Graduada de Trabajo Social Beatriz Lassalle, Universidad de Puerto Rico en Río Piedras
} 
El presente trabajo discute las relaciones del poder masculino, concentrándose en sus implicaciones para el desarrollo de política pública y su impacto en la administración pública. Para ello se discute la idea de lo masculino como referente a la idea de poder y se analiza su alcance e impacto en la gestión pública. Como parte de la temática necesaria para abordar este tema, se reflexiona, además, sobre la situación del hombre sumergido en el quehacer de la política pública en contraposición con las circunstancias del hombre como sujeto de la política pública. Por último, se discuten las implicaciones para la sociedad civil como ente interventor contra las manifestaciones de poder ejercidas por la hegemonía masculina.

\section{El referente masculino del poder}

La historia de la humanidad transcurre entre procesos, relaciones y conflictos diversos a diferentes niveles estructurales que dan paso a las nuevas ideas y necesidades así como a las formas que se responde a éstas a través del proceso de elaboración de política publica. En esa dimensión del quehacer político de los pueblos, las relaciones de poder juegan un rol medular que sirve de base a las acciones que toman las personas, grupos e instituciones y hasta los estados que interesan acceder al poder o, cuando menos, liberarse del yugo del poder que ejercen otras instancias sobre ellos.

La idea del poder está íntimamente ligada al género y a la figura masculina (Ramírez, 1993). Primero, porque se ha concebido la historia moderna imbricada en el eurocentrismo y esto supone que el hombre, en específico el hombre blanco y burgués, es quien domina las esferas de poder (Ani, 1996; Quijano, 2000b). La búsqueda y ejercicio del poder no se limitó a los círculos familiares o territoriales sobre los cuales se tenía dominio, sino que persiguió la expansión y con ella, acrecentó los alcances y dominios de dicho poder. En esos términos relacionados al poder, se da paso al desarrollo e implementación de la primera globalización y se establece la visión del hombre como ente investido de poder y por lo tanto se comporta regido por un discurso masculino de conducta, que al traducirse en códigos, dicta lo que el hombre puede o no puede hacer para cumplir con el estándar del "verdadero hombre", bajo el modelo hegemónico del poder masculino.

Ese "verdadero hombre" encarna y ejemplifica las manifestaciones de la masculinidad hegemónica. Este es el tipo de masculinidad que se establece como norma y que indica al hombre que debe mostrarse siempre como fuerte, inhibirse a la demostración de sensibilidades o emociones de afecto o ternura, siempre estar dispuesto sexualmente, ejercer dominio sobre las mujeres y sobre otros hombres considerados débiles, usar la violencia cuando lo estime necesario y ser proveedor económico de la familia, entre otros atributos. (Kimmel, 1992; Ramírez, 1993; Connell, 1995; Faur, 1995; Valdés y Olavarría, 1998; Ramírez y García, 2002; Lomas, 2004; Huggins-Castañeda, 2005; Nieves-Rolón, 2008; y otros). Ciertamente, la literatura es abundante en la descripción de la masculinidad hegemónica.

La asociación que se hace entre lo masculino igual a fortaleza y poder, se da en contraparte a la idea de lo femenino equiparándolo a debilidad y emocionalidad (Connell, 1995). Por esto es importante que a tales efectos, el hombre actúe y se exprese, validando continuamente su masculinidad y sin dejar espacio para dudas. Lo contrario, sería aceptar públicamente que se es un hombre débil, o se es "poco hombre", y a su vez, implica poner en duda, entre otras cosas, su virilidad e incluso arriesgarse a ser comparado con una mujer. Ésta es otra razón de peso para la urgente necesidad de revisar estas guías y códigos a los cuales se sujetan acríticamente la mayoría de los 
hombres en nuestra sociedad y de la misma forma las instituciones socializadoras como lo son la familia, la iglesia y la escuela, entre otras. En éstas no solo se encierra una visión deformante sobre el hombre, sino que se explica a base de una cultura de inferiorización de la mujer y de los hombres que se aparten del modelo hegemónico imperante.

El asunto del poder y de cómo los hombres lo ejercen no se da únicamente en la relación del hombre con la mujer o con otros hombres considerados más débiles (Ramírez y García, 2002). Es una relación de poder que se da a cualquier instancia, como plantea Foucault (1991), ya sea del padre a la familia, del superior del convento o del gobierno a la gente. Quede claro que la alocución de Foucault trata las relaciones de poder de forma amplia y variada. No obstante, observamos que el asunto del género está implícito en su planteamiento, primero, al observar que el poder al cual se refiere está enmarcado en circunstancias patriarcales y, segundo, sus ejemplos tratan continuamente sobre hombres en el ejercicio del poder.

Estas visiones sobre la masculinidad han sido asimiladas en las tierras dominadas por el expansionismo imperialista. Sin embargo, como cita Lomas (2004), "la masculinidad no es una esencia universal e inalterable sino un efecto de la cultura, un "performance", el lastre histórico y social de una existencia humana conjugada a lo largo de la historia en masculino" (p.12). Se vive masculino, se actúa masculino y, por ende, se es masculino. Ejercer y vivir la masculinidad favorecida por el sistema social hegemónico es un arte que requiere ser cuidadosamente trabajado.

Las características de la masculinidad hegemónica, así como otras características socio-culturales, bajo las cuales se inserta y contextualiza, emergen al interior de la organización de la familia burguesa europea. Esta "nueva organización familiar" estructura las relaciones entre el hombre y la mujer, su asociación procreativa y asigna roles a cada parte (Engels, 2000). Dichas nociones se han impuesto a través del colonialismo y consideradas como organización universalizada hace dos o tres siglos (Meléndez, 2005). Las ideas sobre el género no son las únicas impuestas en el proceso de colonización, sino que traen consigo una agenda de clasificaciones o gradaciones de la población, como "raza" y "clase", entre otras. Estas clasificaciones logran mantener las poblaciones dominadas y a su vez subordinadas, divididas y sometidas al poder opresor, habiendo pasado siglos de dicho acontecimiento (Quijano, 2000a).

En contextos como Puerto Rico, continúan sustentándose y validándose los paradigmas que asignan poder y de la misma forma, aquellas personas que viven las luchas del poder político necesitan conducirse por dichos códigos. Estos códigos a su vez, los llevan a promover y sostener que las estructuras del Estado validen, sustenten y se apoyen en dichos esquemas de poder. Este es el caso de las figuras públicas que, tienen acceso al poder y el dominio político y por ende potestad para elaborar política pública. De modo general, mayormente hombres con poder político que elaboran política pública que sustenta su poder y el de las elites que los apoyan y a su vez oprimen los grupos vulnerados por la sociedad, a través de las propias estructuras del Estado. Preocupa en qué medida las políticas públicas son sujetas a convertirse en este sentido en instrumentos de opresión en manos de quienes dominan el poder político y económico.

\section{Implicaciones de las manifestaciones hegemónico-masculinas en la gestión pública}

Es importante considerar en su justa perspectiva el impacto de la dimensión de género en las dinámicas que se dan entre aquellas personas que tienen la potestad de tomar decisiones políticas 
en las esferas del orden público. Es decir, que las decisiones que se toman, con frecuencia, están matizadas por nociones particulares sobre el género que pueden verse plasmadas en decisiones y acciones vinculadas a familia, orientación sexual, o cualquier aspecto en el que se diferencien las condiciones del hombre y de la mujer. Como ejemplos de relevancia social, encontramos la implantación de un código de vestimenta, la custodia de los hijos e hijas y la adopción.

El hombre ejerce el poder sobre los demás a través de un conjunto de prácticas socioculturales y éstas son justificadas en nombre de la naturaleza o de la razón (Lomas, 2004). La ideología hegemónica se sostiene y se reproduce por medio de las instituciones y desde prácticas que se establecen y ratifican a través de la escuela, religión, artes, política y la cultura en general (Alegría, 2002). Desde esta misma perspectiva las instancias gubernamentales, así como otras privadas, dominadas por esta mirada, son manejadas desde el poder masculino y se convierten en herramienta para institucionalizarlo. No obstante, hay otra área de singular importancia en la que se ven manifiestas las dinámicas de poder basadas en el orden de lo masculino. Estas son las interacciones entre aquellos que tienen dicho poder de toma de decisiones o, bien sea, las personas, de ambos géneros, que ejercen la política.

Los políticos por razón de lo que popularmente se espera de ellos, entran continuamente en dinámicas de juego de poder para lograr su cometido. De hecho, el excesivo peso que tiene el dominio de poder parece convertirse en el objeto central de la política (Tomassini, 1998). Estas circunstancias pueden explicarse a través del modelo de análisis de política pública conocido como la Teoría de Juego (Dye, 2004). Este modelo explica que aquellos que tienen su agenda política, tienen que lograrla y para ello se mueven por diversas vías y los resultados que obtienen dependen de las alternativas seleccionadas por cada cual. Para este modelo es importante la estrategia y considerar que es necesario entrar en dinámicas intra-instancias e inter-instancias para lograr lo propuesto. Al entrar en estas dinámicas hay que considerar un factor concomitante como lo es el género y las circunstancias y contextos ante las cuales se dan las relaciones entre los políticos.

La mayoría de las posiciones de poder político continúan estando en manos de hombres que han asimilado estos discursos de supremacía masculina. Este pensamiento se da unido a que la capacidad y deseo de competir, dominar y triunfar es parte de lo que los hombres asimilan como parte de su identidad masculina (Cazés, 2004). Asimismo, los patrones hegemónicos de masculinidad se asocian incluso a los ideales de fortaleza de identidad nacional (Connell, s.f.). Esto convierte el terreno de lo político en un ruedo constante de competencia por la prevalencia del orgullo personal masculino.

Por otro lado, las mujeres que entran a dichas esferas dominadas por el género masculino se incorporan irremediablemente a las dinámicas de poder institucionalizadas por los hombres para ser aceptadas y legitimadas, llegando algunas a dominar con particular destreza tal juego de poder (Lagarde, 2003). Esto sucede de tal forma debido a que la comparación con el hombre sigue siendo el criterio a partir del cual se mide la ejecución de la mujer en la esfera de lo público (Badinter, 1992). Con frecuencia se observa cómo las mujeres que entran en la arena política asumen para sí los atributos y características de lo masculino para poder competir en igualdad de condiciones a pesar de las diferencias de género, en ocasiones sin tener conciencia de este proceso y sus efectos. Desde esta perspectiva, de modo general, las mujeres terminan apoyando y promoviendo legislaciones y políticas públicas dentro de la óptica de lo masculino. 
Es común ver cómo las circunstancias de los políticos se dan entre confrontaciones y alianzas que lo que persiguen es el poder. Para ello, en muchas ocasiones, los políticos asumen actitudes en las cuales pasa a un segundo plano el bienestar público y prevalece su acción en la permanencia en los puestos que les aseguran el poder o la búsqueda del apoyo del poder político partidista, entre otros. Como comenta Foucault (1991) sobre El Príncipe de Maquiavelo, "el objetivo principal del príncipe debía ser el de conservar su principado" (p.17). Es decir, desde esta perspectiva el objetivo principal de aquellos en puestos políticos no será otro que el de conservar su silla -referente de poder- y para ello se hará lo necesario. Al respecto entendemos que las consideraciones de género pasan a un segundo plano, dándole espacio a los personalismos y metas político partidistas. La relevancia de este particular radica en que, en muchas ocasiones, las actitudes asumidas por políticos están matizadas por los paradigmas de cómo se debe comportar un hombre, según el discurso hegemónico. Desde esta disposición se ejercen prácticas de rivalidad, confrontaciones, pre-potencia, terquedad, asecho y otras formas de violencia, que se distancian por mucho de ser manifestaciones de las diferencias políticas o político partidistas. Lo que está como base de esas conductas no es otra cosa que una demostración de "hombría" y en ese juego, se compromete el bienestar del pueblo.

El análisis de estas manifestaciones de poder por género, es necesario para la comprensión de la dinámica pública y de la gestión de un estado gobernable. Es decir, la gobernabilidad no es un asunto de mera gestión técnica pública. Como plantea Tomassini (1998), el problema de la gobernabilidad no es exclusivamente político, sino que obedece a factores económicos y sociales entre otros aspectos. La cuestión de género es un aspecto social que afecta la ejecución y la efectividad de la gestión pública e incide en las dinámicas que llevan a la ingobernabilidad y es prácticamente norma, que pase de forma inadvertida.

Según plantea, Boeninger (1994), una de las condiciones para la gobernabilidad es la existencia de compatibilidad entre equidad y crecimiento. No obstante, con las implicaciones de la lucha sostenida por los esquemas de poder masculino dicha compatibilidad no necesariamente se hace viable. De manera que pierde sentido la misión de la política pública de servir los necesitados y promover el bienestar de la sociedad.

\section{EI hombre hacedor de política versus el hombre sujeto de la formulación de la política}

Tomando el tema del género como eje central de este artículo, podemos reflexionar sobre la participación de los hombres en materia de política pública. Primero, hay que dejar claro que los hombres han sido los que históricamente han tenido la potestad y el poder como elaboradores de política pública. Esto, debido a que el desarrollo de los gobiernos y la política ha sido un asunto público y según las definiciones patriarcales, lo público está en la esfera de asunto de los hombres. En otras palabras, los hombres han sido tradicionalmente quienes han mantenido el poder de las estructuras políticas, económicas y sociales y, por ende, un dominio casi absoluto de lo público. Por lo tanto, hacer política ha sido mayoritariamente un espacio privilegiado de hombres y de las masculinidades (Ramírez y García Toro, 2002).

Analizando el devenir histórico de los movimientos de sectores oprimidos que han luchado para integrarse en los procesos políticos, es necesario notar la diferencia de que en el caso de los hombres no se ha dado esa lucha por asumir que tienen el poder. A pesar de que el discurso de género tradicional ha visualizado a los hombres como un todo, como un grupo homogéneo, 
es necesario destacar que no todos los hombres tienen poder político. El poder de incidir en lo político ha comprendido tradicionalmente a hombres en posiciones cuyas características los vinculan a grupos hegemónicos. Es importante analizar estas intersecciones a la hora de reflexionar y hacer críticas sobre el poder masculino. Esto es así visto que dentro de la categorización de lo masculino, hay estratos, gradaciones y esferas clasificatorias que asignan mayor poder a unos y a otros asignan subordinación. Es decir, que aun cuando los hombres comparten una ideología hegemónica basada en el patriarcado, la misma ideología se manifiesta de forma diferente de acuerdo a la posición que ocupe el hombre en la jerarquía social (González, 2002). Entonces, al reconocer que no todos los hombres han tenido el poder político en esta esfera, cabe preguntarse qué sucede con esos otros hombres no representados, que en su cotidiano continúan replicando el poder de los hegemónicos pero que para efectos de la política pública, han quedado excluidos y poco representados.

Urge cuestionarse quién cabildea por estos hombres, si desde esta perspectiva se percibe al hombre como poderoso hacedor de política pública y no se ha explorado al hombre común como un ente invisibilizado por estas políticas. Al respecto plantea Faur (1995):

La importante heterogeneidad social de nuestros países, resulta en que la gran mayoría de los hombres se encuentren relativamente desprovistos de poder frente a sus propios congéneres. Esto permitiría desarrollar conexiones entre las políticas de género que desafían el patriarcado y otras políticas que procuran transformar disparidades étnicas o de clase presentes en las relaciones sociales (p.95).

Como sugieren Ramírez y García Toro (2002), los hombres tienen la capacidad de subordinar otros hombres, por lo que no podemos partir de la premisa de que porque se es hombre, se es poderoso por naturaleza.

Es necesario que las políticas sociales sean también observadas a la luz del impacto de las relaciones al interior de los géneros en la vida cotidiana y que se vea cómo las diferencias entre las mujeres y los hombres se convierten en discriminaciones que establecen brechas en la forma de vivir y en el acceso a la calidad de vida (Huggins-Castañeda, 2005). Estas discriminaciones no son sólo contra la mujer, son también, por así decirlo, discriminaciones del hombre contra el hombre, evidenciando de la misma forma la capacidad de subordinación de estos consigo mismos.

Con frecuencia se considera al hombre como el opresor y sin dudar la realidad estructural de dicha cualidad, es necesario sentar las bases de que para transformar dicha condición no basta solamente con el trato al hombre como victimario. Tal consideración hace necesaria una transformación mayor que considere los aspectos estructurales, culturales, sociales e individuales, ya que es en esos espacios donde se desarrollan las subjetividades que dan vida a la sustentación de las opresiones a las que somos sujetos independientemente del género. Visto así, las políticas que quieran proteger a la mujer de la violencia doméstica quedarán irremediablemente inacabadas -siendo simplemente asistencialistas- si no se prevé para la modificación de los contextos y circunstancias que convierten al hombre en agresor y a la mujer en su víctima (Nieves-Rolón, 2008). Urge, desde esta perspectiva, desarrollar políticas que focalicen tanto en los aspectos de género masculino como femenino y que considerando sus especificidades y características peculiares se planifiquen acciones donde se intervenga con los hombres y mujeres que han incurrido en actos 
de violencia doméstica, así como con quienes no. Es necesario que estas intervenciones tomen en cuenta los conocimientos existentes sobre las masculinidades y cómo se prefiguran y configuran culturalmente las relaciones con las mujeres (Olavarría, 2001).

Las luchas por la equidad de géneros deben superar las dicotomías. Más efectivo sería provocar una transformación del sistema a través de cambios de paradigmas, discursos, modelos y códigos, evitando caer en una lucha de poder entre hombres y mujeres. Puerto Rico, aun comienza escasamente a plantearse la situación de los hombres desde la perspectiva de género masculino y en toda América Latina apenas se inician estos debates, por supuesto, centrándose prominentemente en el ámbito de las políticas orientadas a las mujeres (Faur, 2006). Al respecto recomendamos el desarrollo de diálogos y reflexiones críticas entre los géneros que lleven a la comprensión y ponderación en la justa perspectiva de las formas en que éstos se encaran a sí mismos y a los otros. No solo entre géneros, sino también al interior de estos.

La transformación de la política donde se considera un énfasis hacia la perspectiva de género masculino, debe promover la búsqueda de la equidad y la justicia social donde nadie sea considerado superior o inferior a otra persona. Debe ir dirigida a la incorporación de los hombres en los escenarios que han sido relegados y a la educación de las nuevas generaciones para propiciar tratos igualitarios entre los géneros. Se trata de promover el diseño de políticas públicas con orientación crítica a la situación de los géneros en ámbitos laborales, educativos, de salud, de sexualidad, de paternidades y maternidades y de la participación política, entre otros. Se hace necesario superar la mirada fija en la violencia como aspecto de la "naturaleza del género masculino", que resulta no solo sesgada y opresora, sino también insuficiente en la búsqueda de la transformación estructural de las relaciones de género.

Un ejemplo de lo planteado es comenzar por reconocer la situación histórica de opresión que han experimentado las mujeres, reconocer el camino andado y las circunstancias que quedan por trabajar. Según expone Burgos Ortiz (2006), la Organización de las Naciones Unidas (ONU) a través de su secretario Kofi Annan señaló que los retos que enfrentan las mujeres no son problemas sin solución y recomendó siete campos para actuar. El problema es que entre esas siete áreas mencionadas no se toca la figura del hombre desde ninguna perspectiva. No obstante, como hemos discutido previamente, la lucha por la equidad de géneros debe considerar e intervenir tanto con el hombre como con la mujer y reflexionar y analizar las circunstancias del género masculino para que a su vez repercuta en beneficio para la mujer. Al respecto expone Faur (2006): "mientras las políticas tendientes a la conciliación de responsabilidades familiares y laborales se enfoquen de forma prioritaria en las mujeres, difícilmente permitirán un avance sustantivo en la transformación de las desigualdades de género" (p.132). Ciertamente huelga el diálogo entre géneros.

El Estado y la política han sustentado históricamente hasta los días actuales, la visión de que el hombre es útil como proveedor y guerrero. Visión ésta que privilegia el discurso de la masculinidad hegemónica, adjudicando peso máximo a esos dos roles masculinos. Por lo tanto, no parece poner esfuerzos en considerar para el hombre el desarrollo de políticas dirigidas a incentivar su integración en otras dinámicas. Esto es claramente observable al constatar que las políticas y legislaciones relacionadas a familia no reconocen ni estimulan la participación del padre en aspectos como el cuidado de sus hijos e hijas salvo el caso del rol de proveedor económico (Faur, 2006). Por ejemplo, son reducidos los esfuerzos para conciliar las responsabilidades familiares y labo- 
rales, como sería el acceso a licencias por paternidad; o el desarrollo de módulos educativos que cuestionen y se planteen las diferencias de género, pero no sólo cuestionando las inconveniencias de esta aculturación para la mujer, sino ponderar críticamente las dificultades que ello conlleva para el hombre.

Es una realidad en el Puerto Rico actual, que son más las mujeres que se preparan académicamente y con más éxito (Bonilla, López, Cintrón, Ramírez y Román, 2005; Rivera Aponte, Ruiz Mercado y García Toro, 2007) pero pocos se preguntan qué sucede que cada vez menos hombres optan por prepararse académicamente (Rivera Aponte, García Toro y Ramírez, 2006). Esto es representativo de que las políticas no sólo son un sistema de normas y regulaciones, sino una práctica discursiva y social (Birgin, 2003 citado por Faur, 2006). Mientras socialmente se dan una serie de circunstancias que perpetúan la inequidad, las políticas revalidan la situación a través de enfoques asistencialistas o a través de la omisión de políticas necesarias en pro de la equidad de los géneros.

Por esto, es importante que las personas elaboradoras de política pública, revisen y se replanteen los paradigmas y discursos sobre género que subyacen sus creencias y actitudes. Si dicha reflexión no se concreta, continuarán ratificando la inequidad a través de los mecanismos del Estado. Y, ciertamente, mirar las políticas sociales desde otra perspectiva es un ejercicio necesario, pero un primer paso será que los hacedores de política se conciencien de sus propias acciones sustentadas por una visión masculino-hegemónica que deforma la justicia y dificulta las posibilidades de una más efectiva elaboración de políticas públicas y de una administración pública más equitativa.

\section{La sociedad civil y la resistencia al poder hegemónico-masculino}

La mirada que se hace a las políticas públicas y al análisis de la administración pública debe ser una donde se considere el impacto social y económico de sus planteamientos, entre otros aspectos. Por ejemplo, debe considerarse el impacto que tiene su implantación en los diversos y en ocasiones muy particulares estratos de la sociedad. Sin embargo, es frecuente constatar que estos análisis estén orientados sólo por criterios económicos y políticos, desatendiendo así otros aspectos sociales como el género (Rivera Aponte, García Toro y Ramírez, 2006).

El ejercicio de la sociedad civil sobre este aspecto de género, cobra un matiz particular. Por lo regular, las luchas de la sociedad civil se dan con el surgimiento de comunidades o grupos organizados que se identifican como afectados ante determinadas circunstancias y se organizan para tomar acciones proactivas y procurar erradicar o al menos batallar, contra aquello que les atañe y les afecta. Esto lleva a concluir que existe la necesidad de que la sociedad civil intervenga con este particular. No obstante, aunque han existido y existen unas gestiones a estos efectos, hay un gran trecho por recorrer.

$\mathrm{Al}$ ponderar cuál ha sido la gestión de la sociedad civil es notable que en la medida que se identificaba la necesidad de buscar nuevas formas de libertad y participación democrática, surgieron los llamados nuevos movimientos sociales como el de la lucha feminista, por ejemplo. La historia nos confirma que en la medida que se fueron desarrollando movimientos de lucha, éstos fueron apoderándose y comenzando a tener mayor presencia en los procesos políticos y en la elaboración de política pública. Estos grupos comenzaron a cuestionar los órdenes de poder y se lanzaron a la búsqueda de nuevas formas de democratización de las sociedades (Olvera, 1999). En la medida 
en que estos actores o actrices de políticas fueron creciendo, se comenzó a dar mayor importancia a la mirada a esos sectores.

La cuestión de equidad y las implicaciones del género, son asuntos que deberían concernirle a toda la sociedad civil y no meramente porque se vean afectados por un grupo poderoso lejano o cercano a ellos. Como se planteara antes, las dinámicas del género y el poder masculino se dan en cualquier estrato de la sociedad. Es decir, que en las dinámicas internas de esa sociedad civil, se dan transacciones matizadas por el género y asignaciones de roles que de ella se desprenden.

En la mayoría de las muestras de acción de la sociedad civil al respecto, han llevado la voz cantante los sectores feministas. Con el avance de las luchas feministas ocurre un movimiento hacia la consideración de las situaciones que viven las mujeres y cómo las políticas responden a esas situaciones. Asimismo, es posible constatar cómo las mujeres fueron involucrándose en la formulación de las políticas, como entes activos procurando no sólo ser beneficiadas en aras de la equidad y justicia sino, en su carácter de propias hacedoras o cabilderas con ese propósito. No se duda de los grandes logros que han obtenido las mujeres a causa de sus luchas (Burgos Ortiz, 2006). Dichos sectores se fueron diversificando de acuerdo a la variedad de sus postulados y sus perspectivas sobre la figura masculina. Los grupos han coincidido en interpretar que hay unas circunstancias sociales y patriarcales que han llevado a la mujer a experimentar opresión por el hecho de ser mujer, pero esos mismos grupos se han diferenciado en la forma de interpretar otras circunstancias de opresión consideradas como intersecciones al asunto del género (Hooks, 2004).

Otra diferencia es la forma de interpretar la participación del hombre en la lucha por la equidad de género. Algunos grupos han interpretado que los hombres no pueden participar de una lucha pro-mujer por considerar que aquellos grupos de hombres interesados en participar de esa lucha son "lobos disfrazados de ovejas" (Colectivo de ideologías y vivencias de los géneros, 2003). Es decir, que los hombres, por ser hombres y disfrutar de los privilegios que asigna dicha cualidad, están imposibilitados de ser aliados en la lucha por la equidad de género. Otros grupos entienden que los hombres que se cuestionan sus privilegios y están dispuestos a redefinir la masculinidad a base de una vivencia de equidad de género, pueden unirse a dicha lucha. El esquema teórico presentado por Ramírez y García Toro (2002), abre espacios de reflexión al respecto.

Es importante que aquellos grupos que se conciencian y organizan contra las implicaciones de la falta de equidad por razón de género y sus implicaciones en el plano político, no caigan presos de nuevas formas de clasificación y segregación que hacen permanente y en ocasiones insalvables, las diferencias entre hombres y mujeres. Parte de lo que sucede es que con la división interna, los movimientos sociales se debilitan. Entonces, la existencia del Estado como ente sostenedor de la institucionalidad de la hegemonía masculina no evoluciona porque los movimientos sociales tampoco devienen como organizaciones capaces de cuestionar sostenida y consistentemente esa institucionalidad. Como plantea Przeworski (1998) "no sólo el Estado sino también la sociedad civil es débil en las nuevas democracias" (p.85).

La idea de los derechos ciudadanos significa asumir una postura en contra de la autoridad establecida (Shklar, 1997). La misión de la sociedad civil en pro de la equidad de género, requiere que ésta se fortalezca para procurar su meta que sería promover el cambio de los paradigmas sociales sobre el género y lograr modificaciones que impacten la forma de hacer política y, por tanto, en los contenidos explícitos e implícitos de las políticas. Uno de los factores que incide en la gobern- 
abilidad es la afirmación de la ciudadanía frente a la autoridad (Tomassini, 1998). Por esto, urge que la ciudadanía se conciencie sobre la relevancia del asunto de género y exija al poder político conductas manifiestas y desarrollo de medidas que correspondan a esa equidad deseada. En este contexto es necesario denunciar las manifestaciones de los poderosos e intervenir para impactar las actitudes basadas en la hegemonía masculina que asumen los políticos. Siendo así, la gestión pro equidad de géneros y su institucionalización sería una acción concreta, que en unión a muchas otras, rendirían a favor de la gobernabilidad.

Por último, como plantea Shklar (1997):

...no puede haber sentido de justicia sin democracia de la vida cotidiana. La cultura que se requiere para sostener y promover la igualdad de los derechos de la ciudadanía de mocrática depende de la conducta y de la voluntad de los propios ciudadanos. (p.82)

La esencia y la viabilidad del bienestar común quedan a merced de hombres y mujeres que así lo procuren. Los paradigmas permanecerán inalterados en la medida que no se cuestionen sus implicaciones. 


\section{Referencias}

Alatorre Rico, Javier (2002). Masculinidad y las políticas públicas. En Gloria Careaga y Salvador Sierra (Comp.). Debates sobre Masculinidades. México: Universidad Nacional Autónoma de México.

Alegría, Idsa (2002). Ideología y política pública: la representación de las mujeres. En Loida Martínez y Maribel Tamargo (Eds). Género, Sociedad y Cultura. (pp. 160 - 181). Puerto Rico: Publicaciones Gaviota.

Ani, Marimba. (1996). Yurugu: An African-Centered Critique of European Cultural Thought and Behavior. New Jersey: Africa World Press.

Badinter, Elisabeth (1992). XY La identidad masculina. Madrid: Alianza Editorial.

Boeninger, Edgardo (1994). La Gobernabilidad: Un concepto Multidimensional. En Luciano Tomassini (Comp). ¿Qué espera la sociedad del gobierno? (pp. 79 - 94). Santiago: Universidad de Chile.

Bonilla, Víctor; López, Annette; Cintrón, Madelyn; Ramírez, Solángel y Román, Rosa (2005). Feminización de la matrícula de educación superior en Puerto Rico. Cuaderno de Investigación en la Educación, 20. Recuperado de http://cie.uprpr.edu

Burgos Ortiz, Nilsa M. (2006). Desde las esferas privadas y públicas: la participación de las mujeres en las políticas sociales en política social y trabajo social. En Nilsa M. Burgos Ortiz, Ed., Política Social y Trabajo Social, Serie Atlantea 3, pp.65-73. San Juan: Proyecto Atlantea, Universidad De Puerto Rico.

Cazés, Daniel (2004). El feminismo y los hombres. En Carlos Lomas (Comp), Los chicos también lloran: Identidades masculinas, igualdad entre los sexos y coeducación. (pp. 35 - 44). Barcelona: Paidos

Colectivo de Ideologías y Vivencias de los Géneros (2003). Masculino que ninguno: una perspectiva sociopersonal al género, el poder y la violencia. República Dominicana: Centerino, S.A.

Connell, Robert W. (1995). Masculinities. Los Angeles: University of California Press.

Connell, Robert W. (s.f.). Masculinities and masculinity politics in world society. Recuperado de http://irw.rutgers.edu/lectures/connelllecture.pdf

Dye, Thomas R. (2004). Understanding Public Policy. Prentice-Hall, New Jersey.

Engels, Frederick.(2000). El origen de la familia, la propiedad privada y el estado. Recuperado de: http://www.marxists.org/espanol/m-e/1880s/origen/cap2.htm 
Faur, Eleonor (1995). Masculinidades y desarrollo social: las relaciones de género desde la perspectiva de los hombres. Colombia: Unicef Arango Editors.

Faur,Eleonor(2006) Género,masculinidades ypolíticas de conciliación familia-trabajo.Nómadas, 24, 130-141. (Universidad Central - Colombia)

Foucault, Michel (1991). La Gubernamentalidad. En Fernando Álvarez-Uria (Comp), Espacios de poder: Genealogía del Poder (Vol. 6) (pp. 9 - 26). Madrid: Editorial La Piqueta.

García,Víctor I.; Ramírez, Rafael y Solano,Luis.(2004).Paternidades en Puerto Rico.Identidades, $2,(2), 44-68$.

González Armenteros, Juan (2002). Construcción, cuestionamiento y deconstrucción del concepto de masculinidad. En Loida Martínez y Maribel Tamargo (Eds), Género, Sociedad y Cultura (pp.43 - 63). Puerto Rico: Publicaciones Gaviota

Hooks,Bell y otros(2004).Otras inapropiables Feminismosdesdelasfronteras. Madrid: Traficantes de Sueños.

Huggins-Castañeda, Magally. (2005). Género, políticas públicas y promoción de la calidad de vida. Caracas, Venezuela: Instituto latinoamericano de investigaciones sociales (ildis).

Kimmel, Michael (1992). La producción teórica sobre la masculinidad. Nuevos aportes. En Regina Rodríguez (Ed.). Fin de siglo, género y cambio civilizatorio. Ediciones de las mujeres, No. 17, ISIS internacional, 129-138.

Lagarde, Marcela (2003). El feminismo y la mirada entre mujeres. Recuperado de: http://www.e-mujeres.net

Lomas, Carlos (2004). Los chicos también lloran: Identidades masculinas, igualdad entre los sexos y coeducación. Barcelona: Paidos

Meléndez, Héctor (2005). Mercado de culpas ¿Sustituye el mercado al patriarcado? San Juan: Ediciones Mágica.

Nieves-Rolón, Irvyn (2009). La Custodia Compartida: Consideraciones desde la Perspectiva de Género Masculino. En José Toro-Alfonso (Comp.), Lo masculino en evidencia: Investigaciones sobre la masculinidad (pp. 287 - 314). San Juan: Ediciones Puertorriqueñas.

Olavarría, José (2001). Y todos querían ser (buenos) padres: varones de Santiago de Chile en conflicto. Santiago, Chile: FLACSO

Olvera, Alberto (1999). Los modos de la recuperación contemporánea de la idea de sociedad civil. En Alberto Olvera (Comp.), La sociedad civil: de la teoría a la realidad (pp. 11 - 53). México: El Colegio de México. 
Przeworski,Adam (1998). Sociedad Civil.En Adam Przeworski (Comp), Democracia sustentable . Barcelona: Paidos.

Quijano, Aníbal (2000a). Colonialidad del Poder y Clasificación Social. Journal of WorldSystems Research, VI, 2, 342-386.

Quijano, Aníbal (2000b). Colonialidad del poder, eurocentrismo y América Latina. En Edgardo Lander (compilador), La colonialidad del saber: Eurocentrismo y ciencias sociales. Perspectivas latinoamericanas, (pp. 201-242). Argentina: CLACSO.

Ramírez, Rafael. (1993). Dime capitán. Reflexiones sobre la masculinidad. Puerto Rico: Ediciones Huracán.

Ramírez, Rafael y García Toro, Víctor I. (2002). Masculinidad Hegemónica, sexualidad y transgresión. Centro Journal, XIV, 1, 5-25.

Rivera Aponte, Ángel, García Toro, Víctor I. y Ramírez, Rafael (2006). Escolaridad y ocupaciones de la fuerza laboral masculina. Cuaderno de investigación en la educación, 21, 129-147.

Rivera Aponte, Ángel; Ruiz Mercado, Ángel L. y García Toro, Víctor I. (2007). Mujer y Brecha Salarial: Reto del Siglo XXI. San Juan, Puerto Rico: Oficina de la Procuradora de las Mujeres.

Shklar, Judith (1997). Justicia y ciudadanía. En Joelle Affichard y Jean Baptiste De Foucault (Comp), Pluralismo y equidad: La justicia social en las democracias (pp. 77 - 90). Buenos Aires: Ediciones Nueva Visión.

Tomassini, Luciano (1998). Gobernabilidad y políticas públicas. En Raúl Arzúa y Felipe Agüero (Eds.). Fracturas en la gobernabilidad democrática (pp. 31 -64). Santiago: Universidad de Chile.

Valdés, Teresa y Olavarría, José (1998). Masculinidades y equidad de género en América Latina. Santiago de Chile, FLACSO 
